\title{
Traffic Load Computation using Corner Detection Technique in Matlab Simulink Model
}

\author{
Pratishtha Gupta \\ Banasthali Vidyapith \\ Jaipur, India
}

\author{
G.N Purohit \\ Banasthali Vidyapith \\ Jaipur, India
}

\author{
Adhyana Gupta \\ Banasthali Vidyapith \\ Jaipur, India
}

\begin{abstract}
Traffic is the major problem which every country faces because of the increase in number of vehicles throughout the world, particularly in large urban areas.

This document presents a new developed Matlab Simulink model to compute traffic load for real time traffic signal control. Signal processing Blockset and video and image processing Blockset have been used for traffic load computation. The approach used is corner detection operation, wherein, corners are extracted to count the number of vehicles. This block finds the location of the corners, the number of corners, and the corner metric values. The developed model computes the results with greater degrees of accuracy and is capable of being used to set the green signal duration so as to release the traffic dynamically on traffic junctions.
\end{abstract}

\section{Index Terms}

Corner detection, Matlab, Vehicle detection, video and Image processing Blockset.

\section{INTRODUCTION}

This document primarily aims at the new technique of video image processing used to count the traffic load in order to compute green signal duration for the release of that traffic, making the implementation of real time traffic signal control possible reducing the congestion as well as the waiting time of all the road users.

The Video and Image Processing Blockset contains block that perform the Corner Detection. Corner detection is a technique for obtaining image features for object tracking and recognition, especially for three-dimensional objects from two-dimensional images. Hence, Corner Detection method can be performed on traffic images captured using CCTV camera, installed at the desired intersection point. The Corner Detection block finds corners in an image using the Harris corner detection, minimum eigenvalue, or local intensity comparison method. This block finds the corners in the image based on the pixels that have the largest corner metric values. Corner detection block also calculates the corner metric matrix and find corners in images.

In the developed model, two types of images have been used, first is the original traffic image and second image is the cropped image with specified number of vehicles. The corner detection block is applied to find the location of the corners, the number of corners and the corner metric values. To count the traffic load, corners located in the original image and the corners located in the second image are compared.

The remainder of the paper is organized as follows. Section 2 briefly presents the related work. Section 3 briefly describes MATLAB and Video and Image Processing and Signal Processing, being used for the development of this model. Section 4 discusses the algorithm and Section 5 presents the experimental model and results. Section 6 presents a brief comparative study between an existing model [22] and the given proposed model.Section 7 draws the conclusion.

\section{RELATED WORK}

\subsection{Image processing}

Christe et al. [1] discussed in this paper focuses on processing an image pixel by pixel and in modification of pixel neighborhoods and the transformation that can be applied to the whole image or only a partial region.

Kastrinaki et al. in [2] present an overview of image processing and analysis tools used in these applications and we relate these tools with complete systems developed for specific traffic applications.

Kang [3] present the terms corners and interest points are used somewhat interchangeably and refer to point-like features in an image, which have a local two dimensional structure. The name "Corner" arose since early algorithms first performed edge detection, and then analyzed the edges to find rapid changes in direction (corners).

Kim [4] discussed Intensity-based corner detectors, such as the Harris corner, can detect corners in noisy environments, but have inaccurate corner positions and miss the corners of obtuse angles. Edge-based corner detectors, such as the Curvature Scale Space, can detect structural corners, but show unstable corner detection due to incomplete edge detection in noisy environments.

Subri et al. [5] present Corner detection in neural network is based on pattern training sample which trained the network. Corner is detected when there is a similarity between corner chain-code trained pattern and chain code of the line drawing.

Candeias [6] discussed Remote Sensing and Image Processing, spatial, spectral and radiometric resolution of digital images is very important in a lot of applications.

Teixeira et al. [7] present the algorithms input is a cornerness map where a value equal to zero means that the pixel does not correspond to an image corner. The greater the value, the higher the probability of the pixel being a corner. The algorithms output is another image, named label map, where each pixel is labeled as "inside" or "outside"; the pixels 
labeled as "inside" belong to the final solution, i.e. represent the position of image corners.

Fu et al. [8] discussed image processing; better models are needed for both the images and their user. Image models should also be used more extensively in the design of optimal image segmentation and feature extraction procedures.

Lin et al. [9] present Image registration is an important part of the image processing and computer vision. On the basis of analyzing two types of image registration, an automated image registration method was put forward to dealing with image registration with similar transformation.

Nakagaki et al. [10] discussed learning-based algorithms for image restoration and blind image restorations are proposed. Such algorithms deviate from the traditional approaches in this area, by utilizing priors that are learned from similar images. Original images and their degraded versions by the known degradation operator (restoration problem) are utilized for designing the VQ codebooks. The codevectors are designed using the blurred images.

Hachama et al. [11] discussed image registration consists in mapping domains of several images onto a common space and results in some corrections of geometric differences between the images. Most of classical registration techniques rely upon the assumption that there exists a relationship between intensities of images to be registered and that this relationship remains the same all over the image domains.

Hsu et al. [12] present image authentication technique by embedding digital "watermarks" into images is proposed. Watermarking is a technique for labeling digital pictures by hiding secret information into the images.

Goldsmith [13] discussed light microscopy; the spatial transverse resolution is a function of the wavelength and numerical aperture. The depth resolution is another function of these parameters. The factors that enable the detection of fine detail make the sharp focusing of more than a thin slice of the depth in an object impossible.

Davies et al. [14] image processing techniques which, using existing closed-circuit television systems can support both data collection and on-line monitoring of crowds.

\subsection{Traffic load computation}

Bhat et al. [15] discussed Object tracking is used to describe the process of recording movement and translating that movement onto a digital model. Simulink with Video and Image processing Blockset enable to run fast simulations for real-time embedded video, vision, and imaging systems.

Gupta et al. [16] have also proposed a model capable of managing intelligent traffic system using CCTV cameras and WAN. The proposed model will make the traffic signaling dynamic and automatic as well. Besides this, it will generate the dynamic messages for the users on the message boards to avoid congestion, reduce waiting time, pollution control, accident control and vehicle tracking.

Goyal [17] discussed Visual sensor networks (VSNs) have been attracting more and more research attention nowadays. Identifying moving objects from a video sequence is a fundamental and critical task in many computer-vision applications.

Llorca et al. [18] discussed floating car data (FCD) refers to technology that collects traffic state information from a set

Simulink environment provides platform for model-Based Design out of a user-Friendly block diagram environment.

Video and image processing consist of Analysis and Enhancement, Conversions, Filtering, Geometric Transformations, Morphological Operations, Sinks, Sources, Statistics, Text \& Graphics, Transforms and Utilities. of individual vehicles which float in the current traffic. Each vehicle, which can be seen as a moving sensor that operates in a distributed network, is equipped with positioning (GPS) and communication (GSM, GPRS, UMTS, etc.) systems, transmitting its location, speed, and direction to a central control unit that integrates the information provided by each one of the vehicles.

Arora et al. [19] present Fuzzy logic technology has the capability of mimicking the human intelligence for controlling the traffic flow. Fuzzy logic traffic lights control is an alternative to conventional traffic lights control which can be used for a wider array of traffic patterns at an intersection. A fuzzy logic controlled traffic light uses sensors that count cars instead of proximity sensors which only indicate the presence of cars.

Arraigada et al. [20] discussed The use of deformation sensors to measure the deflection of pavement layers due to traffic loads is difficult and often involves complex installation procedures. Experiments summarized in this paper focus on investigating the feasibility of using accelerometers instead of deformation sensors in the road.

Kastrinaki et al. in [21] present an overview of image processing and analysis tools used in these applications and we relate these tools with complete systems developed for specific traffic applications. Image processing also finds extensive applications in the related field of autonomous vehicle guidance, mainly for determining the vehicle's relative position in the lane and for obstacle detection. assembled.

\section{EXPERIMENTAL ENVIRONMENT}

\subsection{Matlab}

MATLAB (matrix laboratory) is a numerical computing environment and fourth-generation programming language. MATLAB allows matrix manipulations, plotting of functions and data, implementation of algorithms, creation of user interfaces, and interfacing with programs written in other languages, including $\mathrm{C}, \mathrm{C}++$, Java, and Fortran. Although MATLAB is intended primarily for numerical computing, an optional toolbox uses the MuPAD symbolic engine, allowing access to symbolic computing capabilities. An additional package, Simulink, adds graphical multi-domain simulation and Model-Based Design for dynamic and embedded systems.

\subsection{Video and Image Processing and Signal Processing}

System objects are algorithms that provide stream processing, fixed-point modeling, and code generation capabilities for use in MATLAB programs. These new objects allow one to use video and image processing algorithms in MATLAB, providing the same parameters, numeric's and performance as corresponding Video and Image Processing Blockset blocks. System objects can also be used in Simulink models via the Embedded MATLAB Function block. Video and Image Processing Blockset blocks support floating-point, integer, and fixed-point data types. To use any data type other than double-precision and single-precision floating point, you must install Simulink Fixed Point.

Signal Processing consist of Estimation, Filtering, Math functions, Quantizers, Signal Management, Signal Operations, Signal Processing Sinks, Signal Processing Sources, Statistics and Transforms. 


\section{METHODOLOGY}

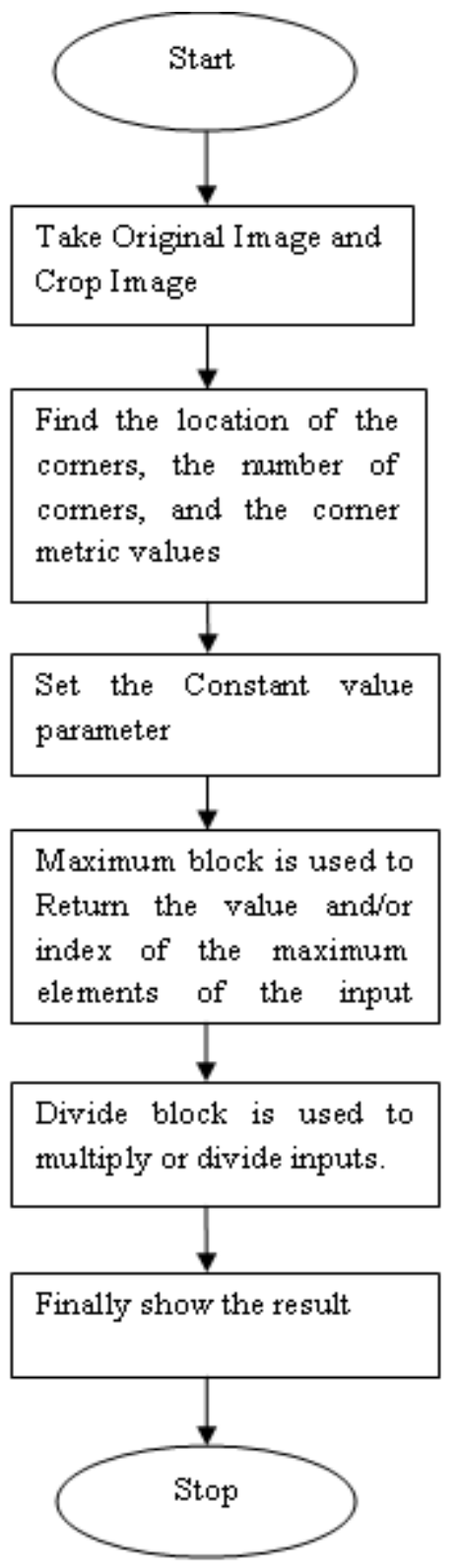

Figure 1:Flow chart for Corner Detection

\section{COUNTING OBJECTS IN AN IMAGE}

The traffic image is imported. Then, using the Corner detection block counted the nearly number of vehicles in the image.

1. Import the Original image from MATLAB using from multimedia file in video and image processing Blockset. (Fig. 2)

2. Import the Crop image from MATLAB using from multimedia file in video and image processing Blockset. (Fig. 3)

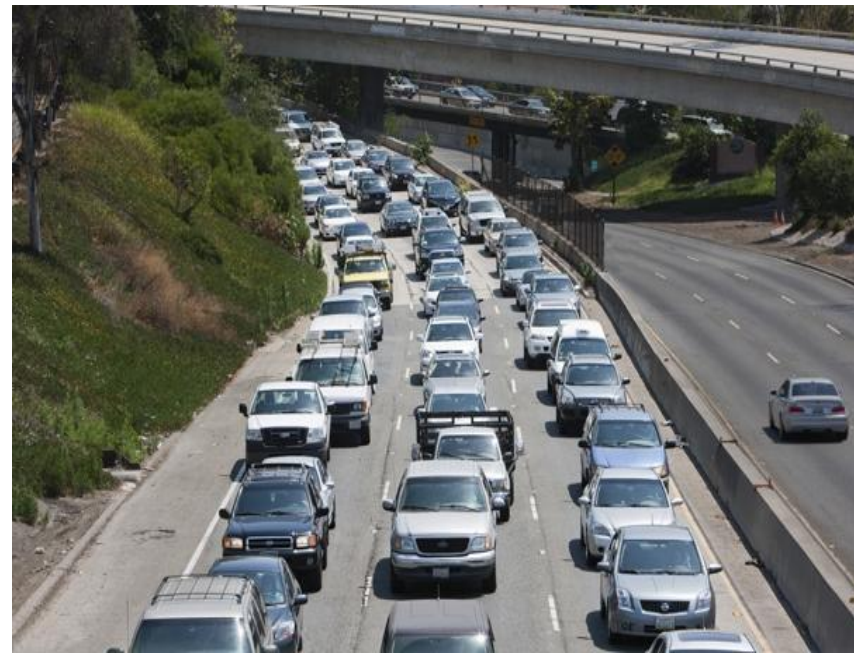

Figure 2:Original image

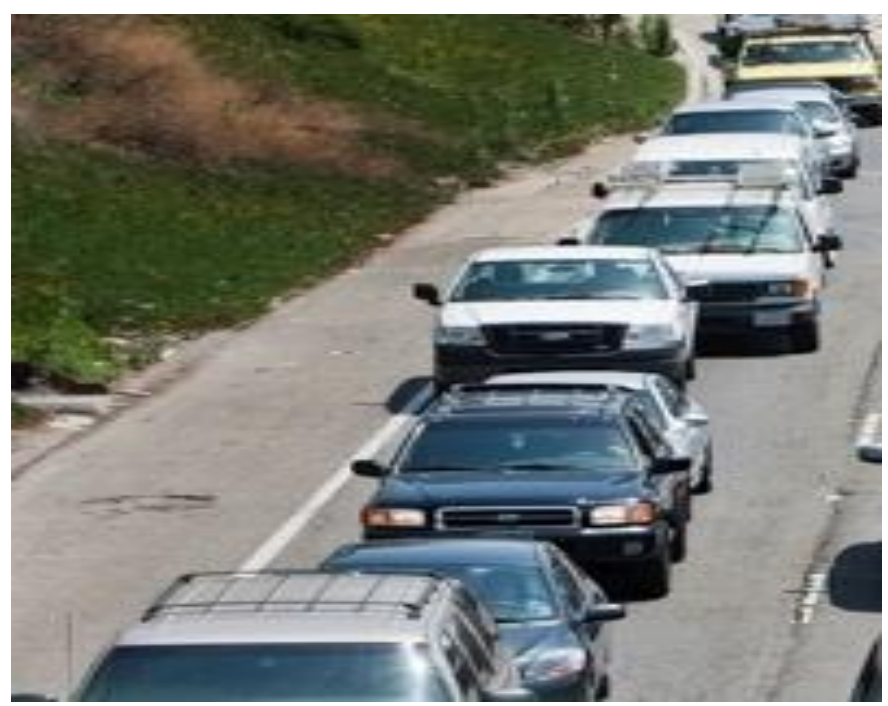

Figure 3:Crop image

3. Create a new Simulink model, and click-and-drag the following blocks into it. (Table 1)

4. The unconnected blocks are arranged as shown in the figure. (Fig. 4)

5. Block parameter are set by double clicking and modifying the block parameters values.

6. Corner block find the location of the corners, the number of corners, and the corner metric values. (Table 2)

7. Set the Constant value parameter. 
Table 1:Blocks Quantity

\begin{tabular}{|l|l|c|}
\hline \multicolumn{1}{|c|}{ Block } & Library & Quantity \\
\hline From Multimedia File & Video and Image Processing Blockset/Sources & 2 \\
\hline Corner Detection & Video and Image Processing Blockset/Analysis and Enhancement & 2 \\
\hline Maximum & Signal Processing Blockset/Statistics & 2 \\
\hline Constant & & 1 \\
\hline Display & Signal Processing Blockset/Signal Processing /Sources & 4 \\
\hline Divide & Signal Processing Blockset/Signal Processing /Sinks & 2 \\
\hline Video Viewer & & 2 \\
\hline
\end{tabular}

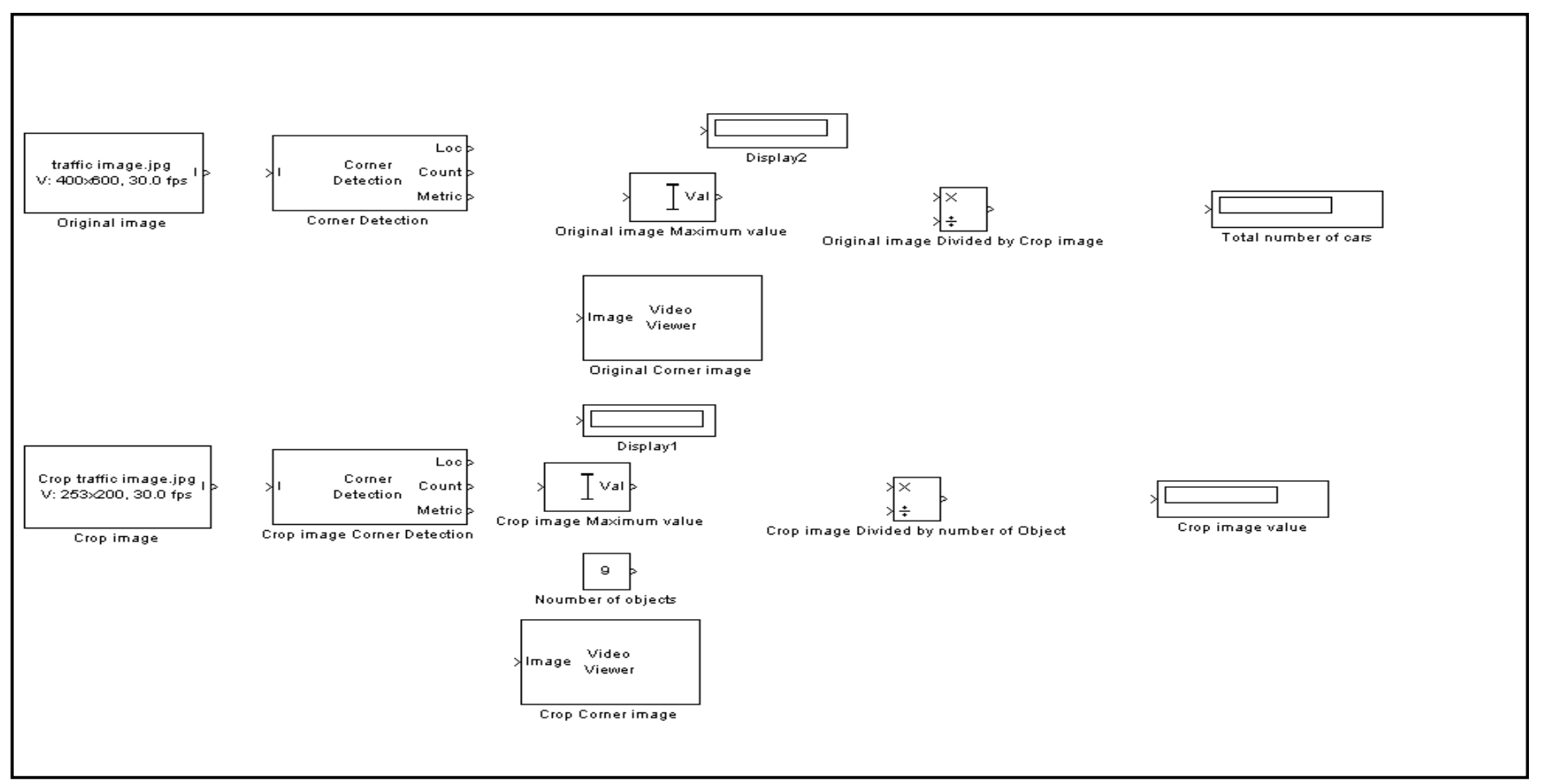

Figure 4:Unconnected Blocks 
Table 2:Corner Detection block parameters set

\begin{tabular}{|c|c|c|c|c|c|c|}
\hline Method & Intensity & $\begin{array}{c}\text { Maximum } \\
\text { Angle } \\
\text { (in degrees) }\end{array}$ & Output & $\begin{array}{c}\text { Maximum } \\
\text { number of } \\
\text { Corners } \\
\text { metric value }\end{array}$ & $\begin{array}{c}\text { Meighborhood } \\
\text { size }\end{array}$ \\
\hline $\begin{array}{c}\text { Rosen \& } \\
\text { Drummond }\end{array}$ & 0.1 & 157.5 & $\begin{array}{c}\text { Corner } \\
\text { location \& } \\
\text { matric matrix }\end{array}$ & 10000 & 0.0005 & {$[1111]$} \\
\hline
\end{tabular}

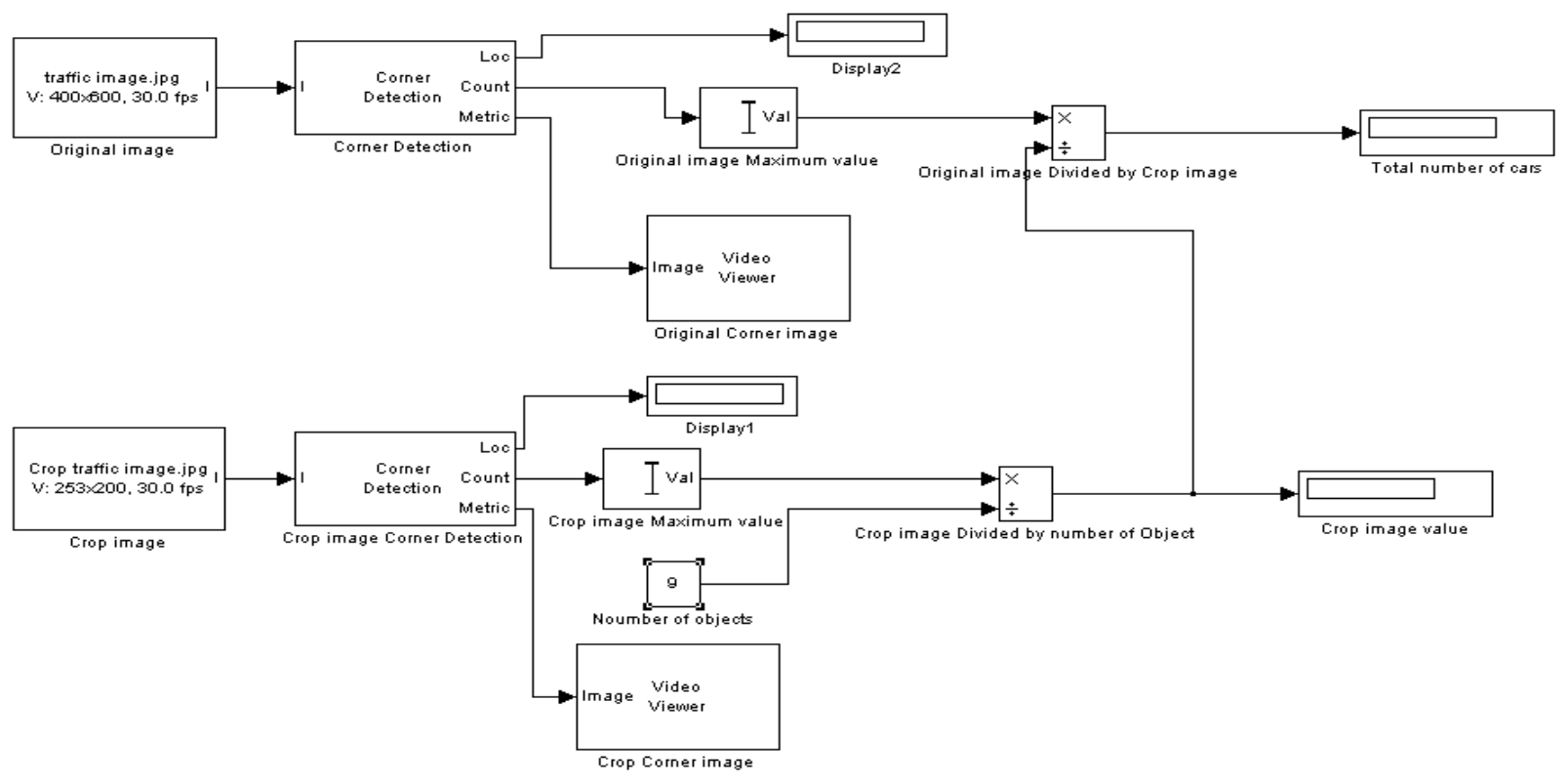

Figure 5:Connected blocks

8. Maximum block is used to Returns the value and/or index of the maximum elements of the input signal.

9. Divide block is used to multiply or divide inputs.

10. Video Viewer block is used to view the Original Corner Image and Video Viewer1 block is used to view the Crop Corner Image.

11. Displays show the number of vehicles in the input image. Use the default parameters.

12. The blocks are connected as shown in the figure.(Fig. 5)

13. The configuration parameters are set. Configuration dialog box is opened by selecting Configuration Parameters from the Simulation menu. The parameters are set as follows:

- Solver pane, Stop time $=0$

- $\quad$ Solver pane, Type = Fixed-step

- $\quad$ Solver pane, Solver $=$ discrete (no continuous states)

\section{Result:}

14. The Original Corner image appears in the Video Viewer window. (Fig. 6)

15. The Crop Corner image appears in the Video Viewer1 window. (Fig. 7)

16. Finally finds the location of the corners, the number of corners, and the corner metric values. (Fig. 8) 


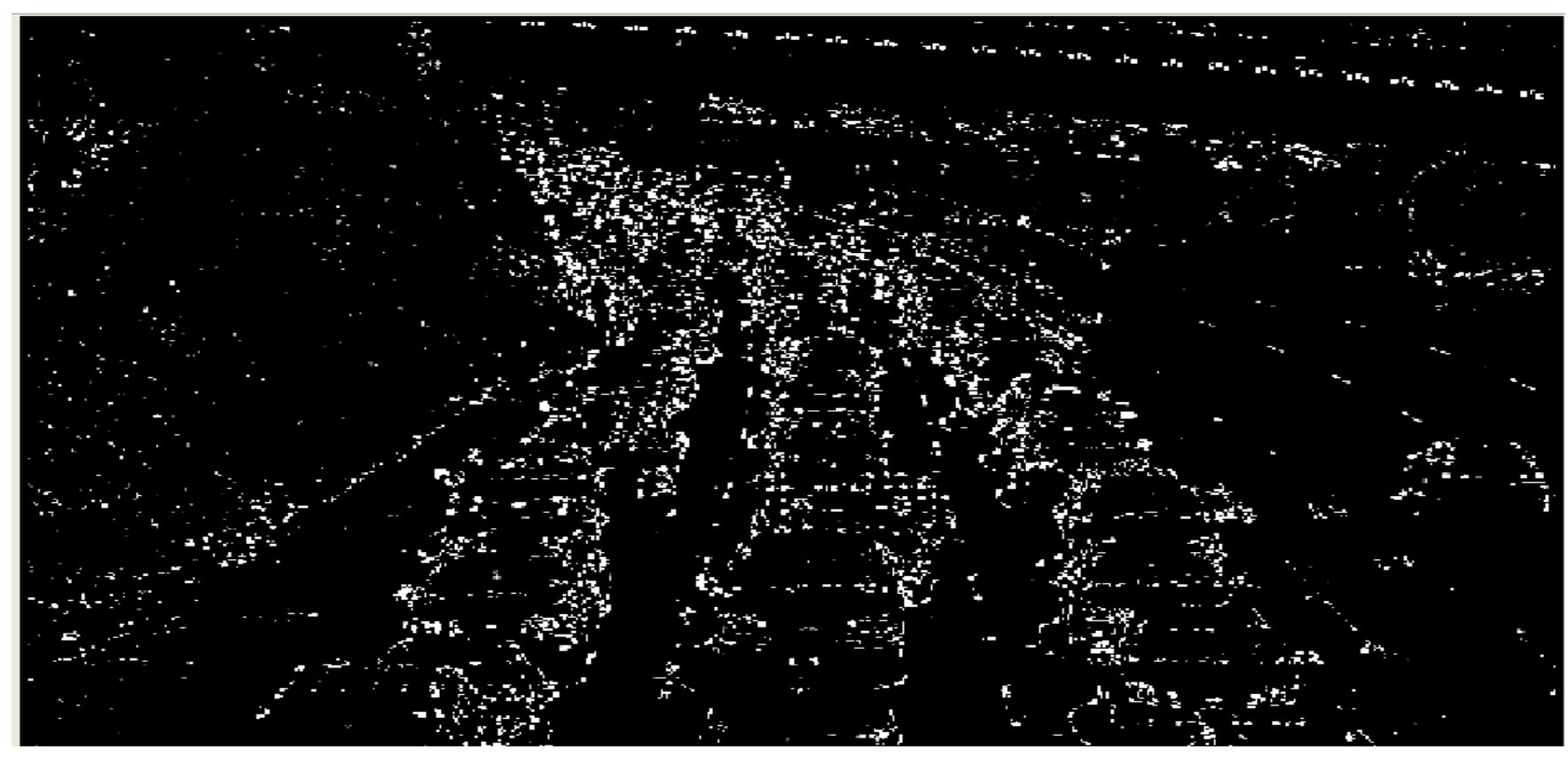

Fig 6: Original Corner image

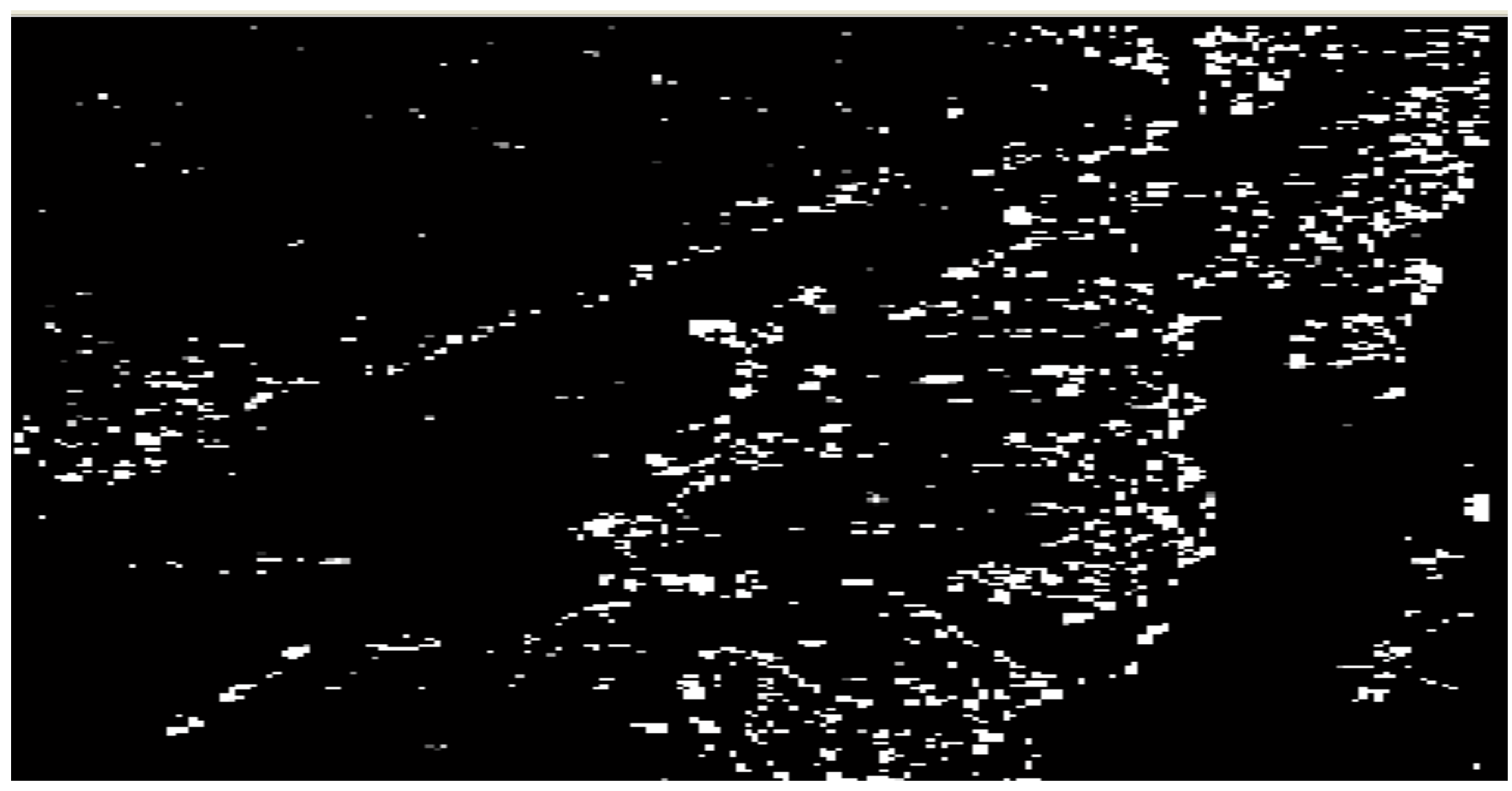

Fig.7: Crop Corner image 


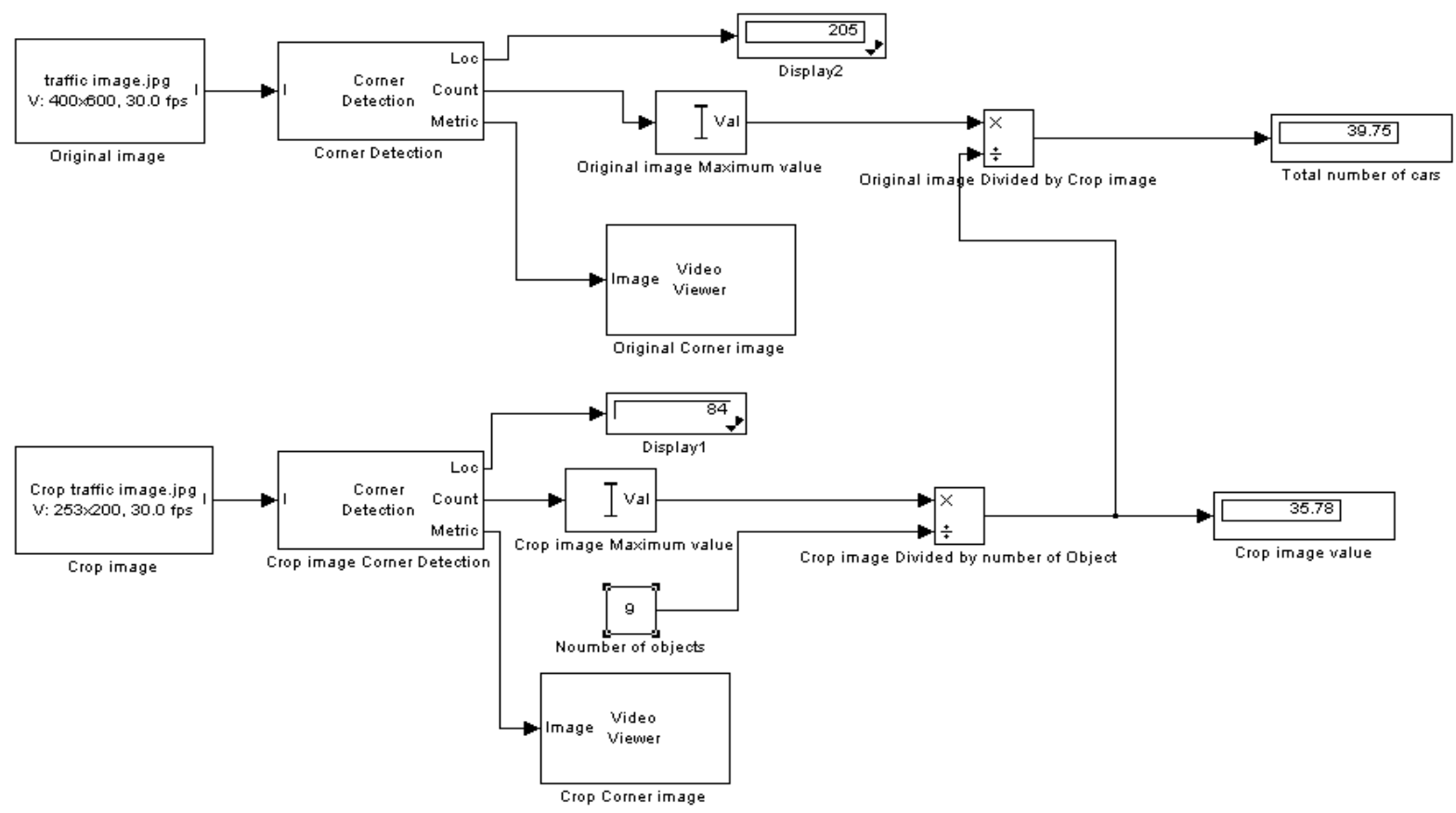

Fig.8: Count number of vehicles (Final Result)

\section{COMPARATIVE STUDY}

Gupta P. et al. in [22] present an another novel approach to compute the traffic load for real time traffic signal control system. A comparative study [table 3 ] is being presented between the model based on edge detection[22] and the proposed model.

Table 3: Comparative Analysis

\begin{tabular}{|l|l|l|}
\hline Comparisons & Model presented in [22] & Proposed Model \\
\hline Approaches & $\begin{array}{l}\text { Edge detection, Histogram } \\
\text { Equalization,Labeling and removing the noise } \\
\text { with the help of median filter. }\end{array}$ & $\begin{array}{l}\text { Signal processing Blockset and } \\
\text { video and image processing } \\
\text { Blockset along with corner detection } \\
\text { operation. }\end{array}$ \\
\hline Better performance & $\begin{array}{l}\text { When the vehicles are placed far apart from } \\
\text { each other }\end{array}$ & $\begin{array}{l}\text { Can compute even when vehicles are } \\
\text { very close to each other and density } \\
\text { is high. }\end{array}$ \\
\hline Input & $\begin{array}{l}\text { Two images of traffic load i.e. one blank } \\
\text { image and one with traffic load are required. }\end{array}$ & $\begin{array}{l}\text { One traffic load image is sufficient } \\
\text { for processing. }\end{array}$ \\
\hline
\end{tabular}




\section{CONCLUSION}

The result shows that the strength of applying corner detection makes corner detector more sensitive in detecting a corner in any image because of the more corners is detected using this method. In this paper, present corner detection technique for traffic load computation. Corner detection technique is very useful for detect the corners in any traffic image. Corner that exists in any irregular line must be detected so that the irregular line can be interpreted to represent actual line. Corners serve to simplify the analysis of images. The developed Simulink model is reliable \& can perform counting the vehicles on roads. This system provides services such as information about the location of the corners, the number of corners, and the corner metric values of the objects. Corner detection methods have a lot scope in future.

\section{REFERENCES}

[1] Christe S.A., Vignesh M., Kandaswamy A., "An efficient FPGA implementation of MRI image filtering and tumour characterization using Xilinx system generator", International Journal of VLSI design \& Communication Systems (VLSICS), Vol.2, No.4, December 2011

[2] Kastrinaki V., Zervakis M., Kalaitzakis K., "A survey of video processing techniques for traffic applications", Image and Vision Computing 21 (2003) 359-381, doi: $10.1016 / \mathrm{S} 0262-8856(03) 00004-0$

[3] Kang B.H., "A Review on Image and Video processing”, International Journal of Multimedia and Ubiquitous Engineering, Vol. 2, No. 2, April, 2007

[4] Kim S., "Robust Corner Detection by Image-Based Direct Curvature Field Estimation for Mobile Robot Navigation", Int J Adv Robotic Sy, 2012, Vol.9, 187:2012, DOI: $10.5772 / 53872$

[5] Subri S. H., Haron H., Sallehuddin R., "Neural Network Corner Detection of Vertex Chain Code", AIML Journal, Volume (6), Issue (1), January, 2006

[6] Candeias A. L. B., "Remote sensing and digital image processing teaching with virtus", The International Archives of the Photogrammetry, Remote Sensing and Spatial Information Sciences, Vol. XXXIV, Part 6, CVI

[7] Teixeira L., Celes W., Gattass M., "Accelerated CornerDetector Algorithms", BMVC 2008 doi:10.5244/C.22.62

[8] Fu K.S., Rosenfeld A.," Pattern Recognition and Image Processing", IEEE transactions on computers, Vol. C25, No. 12, december 1976

[9] Lin H., Du P., Zhao W., Zhang L., Sun H., "Image Registration Based on Corner Detection And Affine Transformation", 2010 3rd International Congress on Image and Signal Processing (CISP2010), 978-1-42446516-3

[10] Nakagaki R., Katsaggelos A.K., "A VQ-Based Blind Image Restoration Algorithm", IEEE transactions on image processing, VOL. 12, NO. 9, september 2003, pp. 1057-7149
[11] Hachama M., Desolneux A., Richard F.J.P., "A Bayesian Technique for Image Classifying Registration", IEEE transactions on image processing, TIP-08569-2012.R1, MAI 2012

[12] Hsu C.T., Wu J.L., "Hidden Digital Watermarks in Images", IEEE transactions on image processing, VOL. 8, No. 1, January 1999, pp. 1057-7149

[13] Goldsmith N.T., "Deep focus; A digital image processing technique to produce improved focal depth in light microscopy", Image Anal Stereol 2000;19:163-167

[14] Davies A.C., Yin J.H., Velastin S.A., "Crowd Monitoring Using Image Processing", IEE Electronic and Communications Engineering Journal, Vol. 7, No. 1 (Feb), pp.37-47

[15] Bhat M., Kapoor P., Raina B.L.,"Application of sad algorithm in image processing for motion detection and simulink blocksets for object tracking", [IJESAT] international journal of engineering science \& advanced technology, Volume-2, Issue-3, pp. $731-736$

[16] Gupta P., Purohit G.N., Dadhich A., "Approaches for Intelligent Traffic System:A Survey", International Journal on Computer Science and Engineering (IJCSE), ISSN : 0975-3397 Vol. 4, No. 09 Sep 2012

[17] Goyal H., "Frame Differencing with Simulink model for Moving Object Detection", International Journal of Advanced Research in Computer Engineering \& Technology (IJARCET), ISSN: 2278 - 1323, Volume 2, Issue 1, January 2013

[18] Llorca D. F. et al., "Traffic Data Collection for Floating Car Data Enhancement in V2I Networks", Hindawi Publishing Corporation EURASIP Journal on Advances in Signal Processing Volume 2010, Article ID 719294 13 pages, doi:10.1155/2010/719294

[19] Arora M., Banga V. K., "Real Time Traffic Light Control System Using Morphological Edge Detection and Fuzzy Logic", 2nd International Conference on Electrical, Electronics and Civil Engineering (ICEECE'2012) Singapore April 28-29, 2012

[20] Arraigada M., Partl M., "Calculation of displacements of measured accelerations, analysis of two accelerometers and application in road engineering", 6th Swiss Transport Research Conference Monte Verita / Ascona, March 15. - 17. 2006

[21] Kastrinaki V., Zervakis M., Kalaitzakis K., "A survey of video processing techniques for traffic applications", Image and Vision Computing 21 (2003) 359-381, doi:10.1016/S0262-8856(03)00004-0

[22] Gupta P., Purohit G. N., Pandey S., "Traffic Load Computation for Real Time Traffic Signal Control", International Journal of Engineering and Advanced Technology (IJEAT), ISSN: 2249 - 8958, Volume-2, Issue-4, April 2013 